\title{
The normoglycaemic biobreeding rat: a spontaneous model for impaired gastric accommodation
}

\author{
Christophe Vanormelingen, ${ }^{1}$ Tim Vanuytsel, ${ }^{1}$ Tatsuhiro Masaoka, ${ }_{1}^{1}$ Gert De Hertogh, ${ }^{2}$ \\ Hanne Vanheel, ${ }^{1}$ Pieter Vanden Berghe, ${ }^{1}$ Ricard Farré, ${ }^{1}$ Jan Tack ${ }^{1}$
}

\begin{abstract}
- Additional material is
published online only. To view please visit the journal online (http://dx.doi.org/10.1136/ gutjnl-2014-308154).

${ }^{1}$ Translational Research Center for Gastrointestinal Disorders (TARGID), University of Leuven, Leuven, Belgium

${ }^{2}$ Department of Pathology, University of Leuven, Leuven, Belgium
\end{abstract}

\section{Correspondence to}

Professor Jan Tack,

Translational Research Center for Gastrointestinal Disorders, University of Leuven, Herestraat 49, Leuven 3000, Belgium; Jan.Tack@med.kuleuven.be

Received 30 July 2014 Revised 17 October 2014 Accepted 22 October 2014 Published Online First 19 November 2014

\section{ABSTRACT}

Objective Impaired gastric accommodation is reported in patients with functional dyspepsia (FD). Previous findings in postinfectious patients with FD suggest that low-grade inflammation and dysfunction of nitrergic nerves play a role in impaired accommodation. To date, spontaneous animal models to study the relationship between these changes are lacking. We hypothesise that the normoglycaemic BioBreeding diabetes-prone (BB-DP) rat provides an animal model of inflammation-induced impaired gastric motor function.

Design Control diabetes-resistant biobreeding, normoglycaemic and hyperglycaemic BB-DP rats were sacrificed at the age of 30,70 and 220 days and gastric fundus tissue was harvested to study nitrergic motor control, inflammation and expression of neuronal isoform of nitric oxide synthase (nNOS) and inducible isoform of nitric oxide synthase (iNOS). Nutrient-induced changes in intragastric pressure (IGP) were measured in normoglycaemic BB-DP rats to study accommodation.

Results No differences in nitrergic function and inflammation were observed between BB-DP and control rats at 30 days. The nitrergic component of the fundic muscle relaxation was reduced in BB-DP rats of 70 and 220 days. This was accompanied by a significant loss of nNOS proteins. IGP significantly increased during nutrient infusion in BB-DP rats of 220 days, indicating impaired accommodation. Infiltration of polymorphonuclear cells, increased myeloperoxidase activity and increased expression of iNOS was observed in the fundic mucosa and muscularis propria of 70-dayold and 220-day-old BB-DP rats.

Conclusions BB-DP rats of 220 days display altered fundic motor control and impaired accommodation, which is least partially explained by loss of nitrergic function. This may be related to inflammatory changes in the neuromuscular layer, suggesting that normoglycaemic BB-DP rats provide a spontaneous model for inflammation-induced impaired gastric accommodation.

\section{INTRODUCTION}

Between meals, smooth muscle in the gastric fundus maintains a high resting tone. Upon food ingestion, a relaxatory response of the proximal stomach occurs, allowing the storage of ingested food without rise in intra-gastric pressure. ${ }^{1}$ This gastric accommodation reflex is mediated by a vago-vagal reflex and involves activation of nitrergic neurons in the myenteric plexus of the proximal stomach. ${ }^{2}$ Nitrergic neurons release nitric oxide

\section{Significance of this study}

What is already known on this subject?

- Several studies reported the presence of low-grade mucosal inflammation in patients with functional gastrointestinal disorders (FGID).

- There is evidence of inflammation at the level of the enteric nervous system and nitrergic dysfunction, being the underlying cause of an impaired gastric accommodation in patients with postinfectious functional dyspepsia.

- These observations suggest interactions between acute inflammation, nitrergic dysfunction and dysmotility in this patient group.

- To our knowledge, there are no spontaneous animal models in which altered gastric accommodation is studied and described.

\section{What are the new findings?}

- The normoglycaemic BioBreeding diabetes prone (BB-DP) rat displayed an impaired gastric accommodation which is partially caused by a loss in nitrergic nervous function.

- This impaired accommodation is associated to low-grade inflammation and loss of neuronal isoform of nitric oxide synthase proteins which develop over time in this model.

How might it impact on clinical practice in the foreseeable future?

- The normoglycaemic BB-DP rat can help us to understand the pathophysiological mechanisms in patients with inflammation-induced impaired gastric accommodation.

- This animal model might also be a useful tool to develop new medications for patients suffering from FGID.

(NO) which induces muscle relaxation. Several observations support a physiological role for the gastric accommodation reflex in determining meal volume and satiation. ${ }^{3}$ Moreover, impaired accommodation has been implicated in the pathogenesis of symptoms in functional dyspepsia (FD), diabetic gastropathy and post-Nissen dyspepsia. ${ }^{4-6}$

FD is a chronic gastrointestinal (GI) disorder characterised by the occurrence of early satiation, postprandial fullness and epigastric pain, in the absence of abnormal findings at endoscopy or other routine examinations. ${ }^{7}$ Impaired gastric accommodation has been reported in
To cite: Vanormelingen
Vanuytsel T, Masaoka T, et al. Gut 2016;65:

$73-81$. 
approximately $40 \%$ of patients suffering from FD. ${ }^{8}$ The mechanism underlying impaired accommodation in FD is incompletely elucidated, but clinical and pharmacological observations have implicated a role for low-grade inflammation and dysfunction of intrinsic nitrergic nerves. The prevalence of impaired accommodation is high $(70 \%)$ in patients with post-infectious FD (PI-FD), and this can be attributed to impaired nitrergic motor control of the proximal stomach. ${ }^{9}$ Patients with PI-FD also maintain signs of ongoing low-grade inflammation up to 12 months after the initiating acute gastroenteritis on mucosal biopsies, with increased number of neutrophils and mast cells, the latter in close proximity to nerve fibres. ${ }^{10}$

These results suggest that dysfunction of nitrergic neurons is a potential underlying cause for impaired gastric accommodation and that this may be triggered by an inflammatory process. This hypothesis is even more plausible, taking into account that infiltration of lymphocytes in the myenteric plexus of the small intestine is associated to neuronal degeneration in patients with IBS. $^{11}$ Additionally, several studies in animal models with induced inflammation in the small intestine showed an impaired neuromuscular function which persists after the initial inflammation and which was characterised by a loss of neuronal nitric oxide synthase (nNOS) expression and a decrease in muscle relaxation and in vivo contractility of the small intestine. ${ }^{12} 13$

Nevertheless, the mechanisms linking low-grade inflammatory changes to impaired nitrergic motor control and decreased gastric accommodation are poorly understood. One reason is the lack of a spontaneous animal model of inflammation-related altered neuronal function and impaired accommodation. Recently, we identified the normoglycaemic BioBreeding diabetes prone (BB-DP) rat as a potential model to study inflammation-induced altered intestinal neuromotor function. ${ }^{14-16}$

The $\mathrm{BB}$ rat is an animal model that has been studied as a model for human type 1 diabetes. This model consists of a control BB-strain (control) and a diabetes-prone strain (BB-DP), of which $50 \%-90 \%$ of the animals will eventually develop diabetes depending on their environment. A combination of prediabetic changes in GI permeability, mucosal inflammation and altered immune function seem to underlie the high prevalence of autoimmune diseases such as diabetes in the diabetes-prone strain. ${ }^{17}{ }^{18}$ It has already been established that diabetic animals show evidence of altered GI neuromuscular function, which has been attributed to consequences of hyperglycaemia. ${ }^{19-21}$

Additionally, several changes in small intestinal neuromuscular function were also reported in normoglycaemic BB-DP rats, including ganglionic inflammation, loss of nNOS expression and nitrergic function. ${ }^{16}$ Studies from our group have linked altered intestinal motility to inflammatory changes in the myenteric plexus. ${ }^{13}$ To date, it remains unclear whether similar events are also present in other parts of the GI tract, including the proximal stomach.

The aim of this study was to evaluate whether the normoglycaemic and hyperglycaemic BB-DP rats develop inflammation in the neuromuscular layer, altered nitrergic nerve function and impaired gastric accommodation. We, therefore, studied the occurrence of inflammation, altered nNOS expression and alterations in neuromuscular function in the proximal stomach of these animals over time. Finally, the effect of these alterations on gastric accommodation was tested in vivo.

\section{MATERIALS AND METHODS \\ Animals}

Breeding pairs of control, normoglycaemic and hyperglycaemic diabetes-prone $\mathrm{BB}$ rats were obtained from the Animal Resources Division of Health Canada (Ottawa, Ontario,
Canada) and further bred in the animal facility of the KU Leuven, Belgium. Rats were housed in wire-meshed cages and had ad libitum access to drinking water and standard rat chow. Rats were sacrificed at different time points $(30,70$ and 220 days) and tissue was harvested. Although our main focus was to study the normoglycaemic diabetes-prone $\mathrm{BB}$ rats (referred to as BB-DP in the remainder of the text), we also added data from age-matched hyperglycaemic diabetes-prone $\mathrm{BB}$ rats (referred to as BB-DPH in the remainder of the text) to find out whether similar changes were present in our cohort. Since diabetes developed in between 90 and 160 days of age, all of our hyperglycaemic animals were sacrificed on day 220 .

\section{Experimental design}

Previous experiments already showed that changes in the small intestine were most pronounced at the age of 220 days. $^{22}$ Based on these findings, changes in intragastric pressure (IGP) during infusion of a nutrient drink were measured in control and BB-DP rats of 220 days old. IGP measurements in BB-DPH animals were not feasible as these animals do not survive the overnight fast that is required for this protocol.

Control and BB-DP rats of 30, 70 and 220 days and BB-DPH rats of 220 days were sacrificed and their stomach was dissected for further research. During dissection of the gastric fundus for muscle strip experiments, fundic mucosa and neuromuscular tissue was harvested for measurement of myeloperoxidase (MPO) activity. The rest of the neuromuscular tissue was preserved for histology, MPO measurements and protein quantification via western blot analysis.

\section{IGP measurements}

Gastric accommodation was assessed through measurements of IGP during infusion of a nutrient drink via an implanted fistula as previously described. ${ }^{23}$ During surgery, under isoflurane sedation, an acryl plastic fistula was inserted into the stomach along the major curvature, opposite to the oesophageal attachment in the stomach. The fistula was then exteriorised through the left abdominal muscle, making it possible to access the stomach lumen from outside the animal. Animals were treated with Baytril (enrofloxacin, Bayer) for 2 weeks in advance and during the entire experiment in order to prevent infections. Rats were also habituated to their restriction cages (Bollmann cages).

After an overnight fast, the rats were placed in Bollmann cages and the fistula was connected to a custom-made infusion system through which a preheated nutrient drink (Nutridrink, Nutricia, Zoetmeer, The Netherlands; $630 \mathrm{~kJ}, 6 \mathrm{~g}$ proteins, $18.4 \mathrm{~g}$ carbohydrates and $5.8 \mathrm{~g}$ lipids per $100 \mathrm{~mL}$ ) was infused. In addition, a polyethylene catheter connected to an external pressure transducer (DPT-6100, Pvb Medizintechnik; Kirchseeon, Germany) was positioned into the stomach through the fistula. IGP was recorded using WINDAQ recording software (DataQ instruments). After a stabilisation period of $30 \mathrm{~min}$, test-meal infusion into the stomach was started at a rate of $30 \mathrm{~mL} / \mathrm{min}$ for $20 \mathrm{~min}$ until a total volume of $10 \mathrm{~mL}$ was infused. The NO inhibitor nitro-L-arginine methyl ester (L-NAME) $(30 \mathrm{mg} / \mathrm{mL} \mathrm{NaCl} 0.9 \%)$ was given subcutaneously 15 min prior to the test meal infusion. L-NAME injection was compared with a control condition where saline $(0.9 \%)$ was injected in the same animal. Changes in IGP were used as a readout for gastric accommodation in the rat stomach as previously reported. ${ }^{23}$ Area under the curve (AUC) of the volumeIGP curve was compared between the different strains and experimental conditions. 


\section{Relaxation experiments with smooth muscle strips} from the gastric fundus

The mucosa of the gastric fundus was removed and muscle strips (length $\pm 10 \mathrm{~mm}$, width $1.5 \mathrm{~mm}$ ) were cut and suspended along their circular axis in an organ bath filled with Krebs $(120.9 \mathrm{mmol} / \mathrm{L}$ $\mathrm{NaCl}, 2.0 \mathrm{mmol} / \mathrm{L} \mathrm{NaH}_{2}-\mathrm{PO}_{4}, 15.5 \mathrm{mmol} / \mathrm{L} \mathrm{NaHCO}_{3}, 5.9 \mathrm{mmol} /$ $\mathrm{L} \mathrm{KCl}, 1.25 \mathrm{mmol} / \mathrm{L} \mathrm{CaCl}_{2}, 1.2 \mathrm{mmol} / \mathrm{L} \mathrm{MgCl}_{2}$ and $11.5 \mathrm{mmol} / \mathrm{L}$ glucose) solution held at $37^{\circ} \mathrm{C}$ and gassed with carbogen $\left(95 \% \mathrm{O}_{2} /\right.$ $5 \% \mathrm{CO}_{2}$ ). After a $40 \mathrm{~min}$ equilibration period at a resting tension of $1 \mathrm{~g}$, acetylcholine $0.1 \mathrm{mmol} / \mathrm{L}$ was added to the organ bath to measure maximal contraction. After a washout period of $30 \mathrm{~min}$, neuronal responses were elicited by electrical field stimulation (EFS), applied via the two parallel platinum rod electrodes using a Grass S88 stimulator (Grass, Guincy, Massachusetts, USA). Frequency spectra $(1-4 \mathrm{~Hz})$ were obtained by pulse trains (pulse $0.35 \mathrm{~ms}$, train $10 \mathrm{~s}, 8 \mathrm{~V}$ ). The voltage was kept at $8 \mathrm{~V}$ by use of a Med Lab Stimu-Splitter II (Med Lab, Loveland, Colorado, USA). In between each pulse train, a $90 \mathrm{~s}$ recovery period was included. The muscle activity was measured using an isometric force transducer/amplifier (Harvard Apparatus, South Natick, Massachusetts, USA), recorded on a multirecorder and sampled for digital analysis using the Windaq Data Acquisition system and a DI-2000 PGH card (Dataq Instruments, Akron, Ohio, USA).

From then on all relaxatory responses induced by the EFS spectrum were performed under non-adrenergic non-cholinergic (NANC, presence of atropine $1 \mu \mathrm{mol} / \mathrm{L}$ and guanethidine $4 \mu \mathrm{mol} / \mathrm{L}$ ) conditions. Precontraction was induced before each spectrum by addition of substance P (SP) $1 \mu \mathrm{mol} / \mathrm{L}$ to the organ bath, which generated a stable tone. Responses were characterised pharmacologically by repeating the spectrum in the presence of L-NAME $(0.3 \mathrm{mmol} / \mathrm{L})$, which was added $15 \mathrm{~min}$ before each EFS spectrum. Addition of L-NAME was used to study alterations in the nitrergic component of the muscle relaxation. Muscle responsiveness to NO was tested by addition of nitroglycerine (NG) $10 \mu \mathrm{mol} / \mathrm{L}$, immediately after the last EFS.

Similar to this protocol, an additional control experiment was performed to compare the effect of the mucosal stripping on the muscle contractility. Muscle strips with and without mucosa coming from the same animal (three in total) were mounted in the organ bath. Subsequently, we tested the muscle response to EFS in the presence and absence of L-NAME as previously described.

The responses to EFS were calculated as the AUC during the stimulation period (on-response) and corrected for the crosssectional area $\left(\mathrm{g} /\left(\mathrm{mm}^{2} \mathrm{~s}\right)\right)$. The percentage decrease of electrical induced muscle relaxation by L-NAME was calculated as $\left(\mathrm{R}_{\text {before }}-\mathrm{R}_{\mathrm{after}}\right) \times 100 / \mathrm{R}_{\text {before}}$, where $\mathrm{R}_{\text {before }}$ and $\mathrm{R}_{\mathrm{after}}$ represent the response before and after addition of the pharmaceutical compound. This method was previously used by Smits et al. ${ }^{24}$

\section{MPO activity}

Fundic tissue was blotted dry, weighed and then minced on ice in a potassium phosphate buffer $(\mathrm{pH} 7.4)$ containing $0.5 \%$ hexadecyltrimethylammoniumbromide (HTAB, Sigma Chemicals, St. Louis, Missouri, USA). For further extraction, the tissue was then homogenised for $4 \mathrm{~min}$ in the same buffer followed by two freeze-thaw cycles. Samples were then centrifuged and $100 \mu \mathrm{L}$ of the supernatant was added to $2.9 \mathrm{~mL}$ of phosphate buffer containing $0.53 \mathrm{mmol} / \mathrm{L} \mathrm{O}$-dianisidine and $0.15 \mathrm{mmol} / \mathrm{L}$ hydrogen peroxide $\left(\mathrm{H}_{2} \mathrm{O}_{2}\right)$. The oxidation of the $\mathrm{O}$-dianisidine is catalysed by the peroxidase present in inflammatory cells, mainly neutrophils. The change in absorbance at $460 \mathrm{~nm}$ was measured every $30 \mathrm{~s}$ for $20 \mathrm{~min}$ in a spectrophotometer (Genesys 6, Thermo LabSystems, Belgium). One unit of MPO activity was defined as the amount of MPO activity required to convert $1 \mu \mathrm{mol}$ of $\mathrm{H}_{2} \mathrm{O}_{2}$ to $\mathrm{H}_{2} \mathrm{O}$ per minute at $25^{\circ} \mathrm{C}$, and activity was presented as units per milligram of tissue.

\section{Histology and immunohistochemistry}

Transmural fundic tissue $\left( \pm 15 \mathrm{~mm}^{2}\right.$ surface area) was fixed in $4 \%$ neutral-buffered formalin, embedded in paraffin and stained with standard H\&E. The biopsies were checked for mucosal damage (presence of erosions or ulcers) and then evaluated for numbers of polymorphonuclear (PMN) cells in the submucosa (total amount of PMN cells per 10 high-power fields $(\mathrm{HPF}),=\times 400$ magnification). The infiltration of PMN cells in the myenteric plexus was also quantified by counting the PMN cells present in the ganglia. Counts were performed by a single experienced pathologist (GDH) who was not informed on animal treatment, symptoms or other experimental data. Connective tissue mast cells were observed after toluidine blue staining and quantified by counting $10 \mathrm{HPF}$ at the intramuscular level (×400 magnification).

\section{RT-PCR analysis}

RT-PCR was performed using a Lightcycler 480 (Roche Applied Science, Penzberg, Germany). LightCycler 480 SYBR Green 1 Master Mix (Roche Applied Science) was combined with cDNA samples and primers for nNOS and iNOS (forward: ACCCAAGGTCTACGTTCAAGACA; reverse: CACATCCCG AGCCATGC) from TIB molbiol, Berlin, Germany. Different $5^{\prime}$-splice variants of nNOS have been reported by other groups, PCR was performed on $\mathrm{nNOS} \alpha$ (three different splice variants $\mathrm{nNOS} \alpha-\mathrm{a}, \mathrm{nNOS} \alpha-\mathrm{b}$ and $\mathrm{nNOS} \alpha-\mathrm{c}$ ) and $\mathrm{nNOS} \beta$ (primer design based on previous literature). ${ }^{25}$ Expression was normalised to the hypoxanthine phosphoribosyltransferase gene, and results are presented as fold change as described previously. ${ }^{26}$

\section{Western blot analysis}

Fundic neuromuscular tissue was dissected and snap-frozen in liquid nitrogen and stored at $-80^{\circ} \mathrm{C}$. Frozen tissue was homogenised in lysis buffer (Tissue Protein Extraction Reagent) and phosphatase inhibitor cocktail (Thermo Fisher Scientific, Rockford, Illinois, USA). After centrifugation, the total protein concentration was determined. Equal amounts of protein $(25 \mu \mathrm{g})$ were run on a 4\%-12\% gradient sodium dodecyl sulfate-polyacrylamide gels (Invitrogen, Carlsbad, California, USA) and then transferred to polyvinylidene difluoride membranes. After blocking with dried milk dissolved in TBS-T buffer (Tris-Buffered Saline and Tween 20), blots were incubated overnight at $4^{\circ} \mathrm{C}$ with the primary antibodies rabbit anti-nNOS (1/200, Santa Cruz Biotechnology, Santa Cruz, California, USA), iNOS (1/200, Abcam, Cambridge, UK), the general neuronal marker: rabbit anti-PGP9.5 (1/500, Merck Millipore, Darmstadt, Germany) and rabbit antiglyceraldehyde-3phosphate dehydrogenase (GAPDH) (1/500, Abcam, Cambridge, $\mathrm{UK})$. Then blots were incubated for $2 \mathrm{~h}$ at room temperature with the horseradish peroxidase-conjugated secondary antibodies raised against rabbit immunoglobulin G. Bands were visualised by incubating the blot for $5 \mathrm{~min}$ in Supersignal West Femto Maximum Sensitivity Substrate (Thermo Fisher Scientific, Doornveld, Belgium). After scanning, bands were quantified by densitometry using Image J software (National Institutes of Health; http://rsb. info.nih.gov.ij/). Expression of PGP9.5 was quantified relative to the housekeeping protein GAPDH. The total amount of nNOS protein was expressed relatively to PGP9.5.

\section{Statistical analysis}

Data are expressed as mean $\pm S E M$, with ' $n$ ' indicating the number of rats. Analysis of the in vitro contractility data was 
done with SAS for Microsoft Windows, V.9.2 (SAS Institute, Cary, North Carolina, USA). Control and BB-DP groups were compared with a mixed model analysis (proc mixed). Strain (BB-DP and control), frequency of EFS (1-4 Hz) and a strain by frequency interaction term were entered in the model as fixed effects. EFS frequency was also included as a repeated effect. Analysis of all other data was performed using Graphpad software (Graph Pad Software, San Diego, California, USA). Differences between two groups were tested by two-tailed unpaired Student $t$ test or Mann-Whitney $U$ test depending on the distribution of the data, which was checked by a Kolmogorov-Smirnov test. Differences between more than two groups were evaluated by analysis of variance with post hoc $t$ test and Bonferroni correction for multiple testing or KruskalWallis test with Dunn's multiple comparison test when appropriate. Results were considered significant with $\mathrm{p}$ value $<0.05$.

\section{RESULTS}

\section{Gastric accommodation}

Changes in IGP during nutrient infusion in the stomach of 220-day-old rats were used as a readout for gastric accommodation to the infused liquid meal, with higher pressures reflecting impaired accommodation. ${ }^{23}$ The IGP during nutrient infusion in BB-DP rats was significantly higher compared with agematched control rats (AUC during nutrient infusion: 105.8 \pm 5.7 vs $80.3 \pm 8.8 \mathrm{~mL} \times \mathrm{mm} \mathrm{Hg}, \mathrm{p}<0.05$ ) (figure $1 \mathrm{~A}$ ). The effect of L-NAME treatment on IGP changes in BB-DP and control animals was compared with saline treatment in the same animals. This was done to evaluate whether the difference in IGP between both animal groups could be attributed to impaired nitrergic function. A significant increase of the IGP occurred when control animals were treated with L-NAME (AUC $68.9 \pm 7.7$ vs $116.4 \pm 13.8 \mathrm{~mL} \times \mathrm{mm} \mathrm{Hg}, \quad \mathrm{p}<0.01$ ) (figure 1A). In contrast, the IGP in BB-DP animals of 220 days did not change when treated with L-NAME (AUC 105.8 \pm 5.7 vs $98.1 \pm 11.0)$. There were no significant differences when comparing the IGP of L-NAME-treated control rats with salinetreated BB-DP rats. The total stomach weight was comparable between both groups. Together, these data demonstrate impaired accommodation in the BB-DP BB-rat, which is at least, in part, attributable to loss of nitrergic neuronal function. The onset of nitrergic dysfunction was studied using muscle strip studies at variable ages.

\section{Neuromuscular function of the gastric fundus}

EFS-induced muscle strip relaxation under NANC conditions was comparable in 30-day-old and 70-day-old BB-DP and control rats (figure 2A, B). At the age of 220 days, relaxation over all frequencies was significantly impaired in BB-DP and BB-DPH rats $(p<0.05)$ (figure $2 \mathrm{C}$ ). Both contraction induced by SP and the total percentage of relaxation induced by NG were the same in all animal groups at all time points, indicating that there were no differences in contractile and relaxatory capabilities of the muscle.

To evaluate potential differences in nitrergic contribution to the muscle relaxation, the L-NAME-induced reduction in muscle relaxation under NANC conditions was calculated. There were no differences in nitrergic contribution at the age of 30 days. In contrast, the nitrergic contribution to the muscle relaxation was significantly smaller in 70-day-old and 220-day-old BB-DP and BB-DPH rats (figure 3).

Control experiments showed that muscle relaxation and the effect of L-NAME on the relaxation was similar when comparing muscle strips with and without mucosal tissue. This confirms that removal of the mucosa has no relevant effect on the muscle strip function (see online supplementary figure S1).

\section{MPO activity in the gastric fundus}

MPO activity was measured in the mucosa and neuromuscular layer of control animals and compared with age-matched BB-DP rats. Mucosal MPO activity at 30 days was negligible and similar in both strains $(0.01 \pm 0.0$ vs $0.0 \pm 0.0 \mathrm{U} / \mathrm{mg} ; \mathrm{p}=0.2)$. In contrast, MPO activity in the mucosa of BB-DP rats was significantly increased at 70 days $(1.10 \pm 0.04$ vs $0.03 \pm 0.01 \mathrm{U} / \mathrm{mg}, \mathrm{p}<0.01)$ and in BB-DP and BB-DPH rats of 220 days (control, 0.02 \pm 0.01 vs BB-DP, $0.63 \pm 0.40, \mathrm{p}<0.01$ and $\mathrm{BB}-\mathrm{DPH}, 0.68$
Figure 1 In vivo measurement of intragastric pressure (IGP) changes. (A) Area under the curve (AUC) of the pressure-volume curve during nutrient drink infusion. Comparison between control (black bars) and BioBreeding diabetes-prone (BB-DP) (white bars) animals was done under saline and nitro-L-arginine methyl ester (L-NAME) treatment. (B) Individual tracing of control animals. (C) Individual tracing of BB-DP rats. (D) Representative tracing of IGP changes by infusion of nutrient drink comparing

L-NAME-treated control animals with BB-DP rats under control conditions. ${ }^{* *} p<0.01$ control saline versus control L-NAME and ${ }^{\gamma} p<0.05$ control saline versus BB-DP saline.
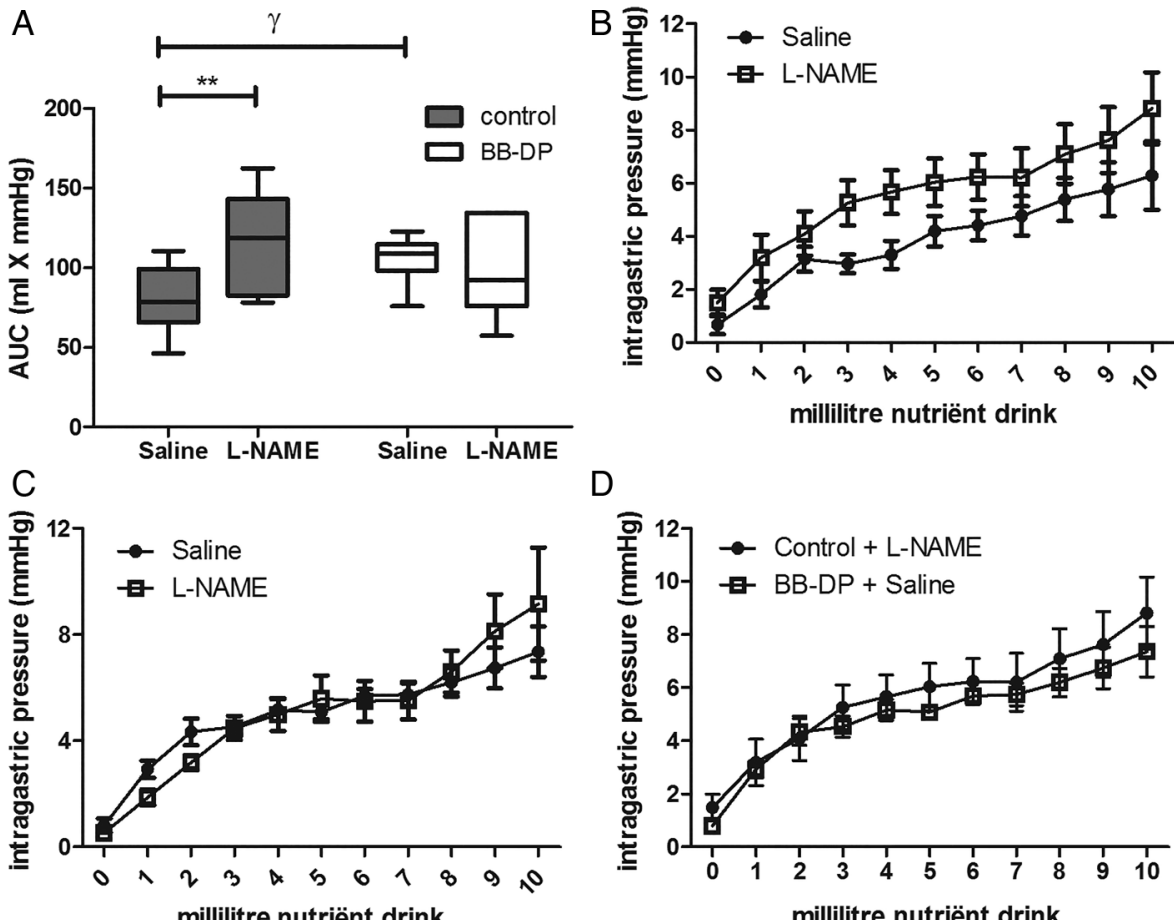

D

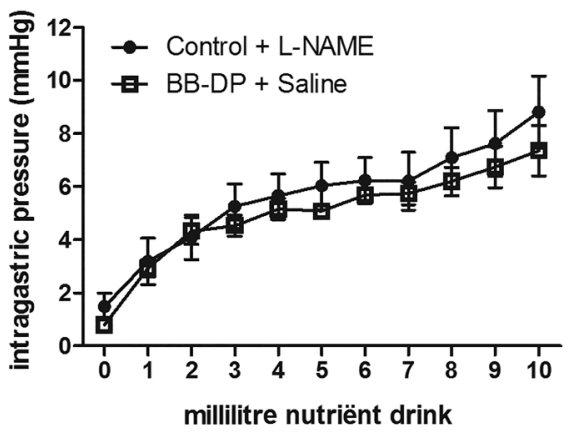



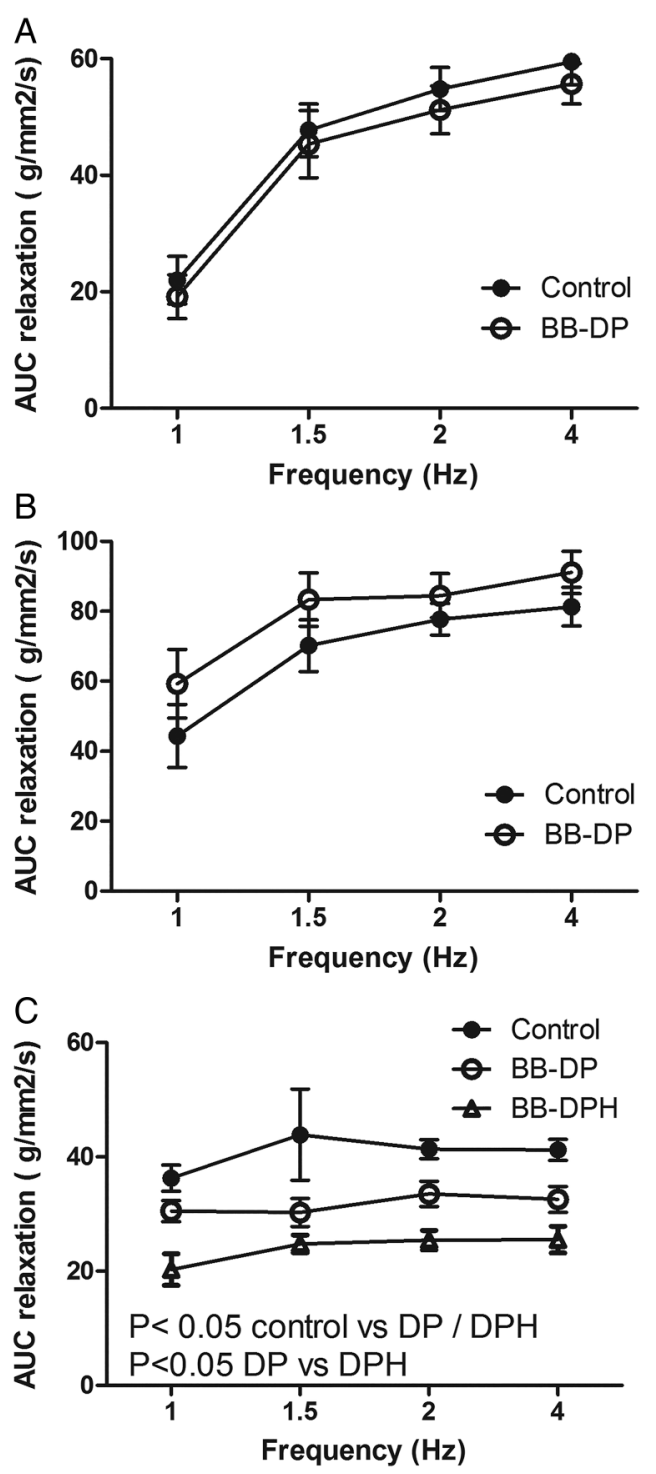

Figure 2 Measurement of EFS-induced muscle relaxation under non-adrenergic, non-cholinergic conditions. Fundic muscle strips were incubated with atropine and guanethidine 15 min before precontraction with substance P. Muscle strip relaxation was induced by EFS stimulation at four different frequencies. Relaxation values were expressed as area under the curve (AUC), which was corrected by the cross-sectional area. Statistical analysis was done by a mixed model analysis, where the main effect of group was studied at the three different ages (N: 6-7; (A) 30 days; (B) 70 days; (C) 220 days). BB-DP, BioBreeding diabetes-prone; BB-DPH, hyperglycaemic diabetes-prone $\mathrm{BB}$ rats; EFS, electrical field stimulation.

$\pm 0.25 \mathrm{U} / \mathrm{mg}, \mathrm{p}<0.05$ ) (figure $4 \mathrm{~A}$ ). This was also the case when MPO activity was measured in the neuromuscular layer of BB-DP rats at 70 days (BB-DP, $0.85 \pm 0.70$ vs $0.01 \pm 0.01 \mathrm{U} / \mathrm{mg}$, $\mathrm{p}<0.01$ ) and in BB-DP and BB-DPH rats of 220 days (control, $0.01 \pm 0.005$ vs BB-DP, $1.00 \pm 0.28, \mathrm{p}<0.01$ and BB-DPH, 0.58 \pm 0.32 units $/ \mathrm{mg}, \mathrm{p}<0.01$ ) (figure $4 \mathrm{~B}$ ).

PMN infiltration in the submucosa and myenteric ganglia

None of the animals displayed any erosions or damage at the mucosal and muscular level when evaluating the gastric fundus macroscopically and microscopically. Absence of PMN cells in the mucosa and myenteric ganglia of both BB-DP and control rats at the age of 30 days confirmed that there was no inflammation at this time point. There was, however, a significant increase of PMN cells in the submucosa of 70-day-old and 220-day-old BB-DP and 220-day-old BB-DPH rats compared with age-matched control animals (figure 5A, C, E). The amount of PMN cells in the myenteric ganglia was also significantly increased in BB-DP rats of 70 and 220 days and BB-DPH rats of 220 days (figure $5 \mathrm{~B}, \mathrm{D}, \mathrm{F}$ ).

The total amount of connective tissue mast cells was significantly increased at $70(3.7 \pm 2.7$ vs $27.2 \pm 14.2 / 10 \mathrm{HPF}, \mathrm{p}<0.05)$ and 220 days (control, $3.3 \pm 1.8$ vs BB-DP, 58.6 $\pm 13.2, \mathrm{p}<0.01$ and BB-DPH $10.4 \pm 2.7 / 10 \mathrm{HP}, \mathrm{p}<0.01)$ in $\mathrm{BB}$ rats, but no differences were found at the age of 30 days.

To investigate whether there is a direct relationship between inflammatory changes and altered gastric function, IGP values of BB-DP rats were correlated with the levels of MPO activity and the amount of PMN cells. Both MPO activity $\left(r^{2}=0.39\right)$ and amount of PMN cells $\left(r^{2}=0.089\right)$ did not significantly correlate with the IGP values of the BB-DP animals.

\section{nNOS and iNOS mRNA expression in the neuromuscular layer of the gastric fundus}

Expression of iNOS and nNOS splice variants was not altered when comparing control rats with both BB-DP and BB-DPH rats. Expression data are summarised in table 1.

\section{nNOS and iNOS protein expression in the neuromuscular} layer of the gastric fundus

The amount of nNOS protein in the neuromuscular layer was expressed relative to the general neuronal marker PGP9.5. No differences in GAPDH and PGP9.5 expression were found at any time point, suggesting that the total amount of neurons was largely preserved. nNOS protein expression did not differ when comparing BB-DP with control rats at the ages of 30 and 70 days (figure $6 \mathrm{~A}, \mathrm{~B}$ ). In contrast, a significant loss of both nNOS and a significant increase of iNOS protein expression occurred in BB-DP $(\mathrm{p}<0.01$ and $<0.05)$ and BB-DPH rats $(p<0.01$ and $<0.05)$ at the age of 220 days (figure 6C). No significant difference was found when comparing nNOS and iNOS protein expression between BB-DP and BB-DPH rats.

\section{DISCUSSION}

Clinical observations and pathophysiological studies suggest that dyspeptic symptoms and impaired gastric accommodation may occur as a consequence of GI inflammation, leading to impaired nitrergic nerve function in the proximal stomach. Unravelling the pathophysiological cascade underlying these events would benefit from the availability of a spontaneous animal model of impaired accommodation and gastric nitrergic dysfunction. In the present study, we demonstrated the occurrence of increased inflammation in the gastric fundus of the normoglycaemic BB-DP rat, followed by impaired nitrergic motor control and decreased expression of nNOS protein. This was accompanied by impaired gastric accommodation, which was at least, in part, attributable to a loss of muscle relaxation induced by the nitrergic neurons. In addition, we found that the same alterations were present in the BB-DPH rat, which is in line with the previous observations in hyperglycaemic BB rats. ${ }^{20}$

Previous studies have reported inflammatory changes in the GI tract of the BB-DP rat. Early data found a marked inflammatory infiltrate in the colon of these animals, both in the muscularis and the enteric ganglia. ${ }^{27}$ Also in the small bowel of these animals, increased MPO activity was reported, which increased over time. ${ }^{28}$ Based on these findings, we studied the occurrence of inflammation in the gastric fundus from control, BB-DP and BB-DPH animals at several time points and measured MPO and 
Figure 3 Nitrergic contribution to the muscle relaxation. Fundic muscle strips were incubated with atropine, guanethidine and nitro-L-arginine methyl ester (L-NAME) for $15 \mathrm{~min}$ before precontraction with substance P. Muscle strip relaxation was induced as previously mentioned. Delta L-NAME was calculated by subtracting relaxatory values of each strip under non-adrenergic, non-cholinergic (NANC) from NANC+L-NAME conditions, after which this delta was expressed as percentage of the relaxation under NANC conditions. Statistical analysis was done by a mixed model analysis, where the main effect of group, comparing control (black dots), BioBreeding diabetes-prone (BB-DP) (white dots) and hyperglycaemic diabetes-prone $B B$ rats (BB-DPH) (white triangles) conditions, was studied at the three different ages (N: 6-7; (A) 30 days; (B) 70 days; ( $C$ and D) 220 days).
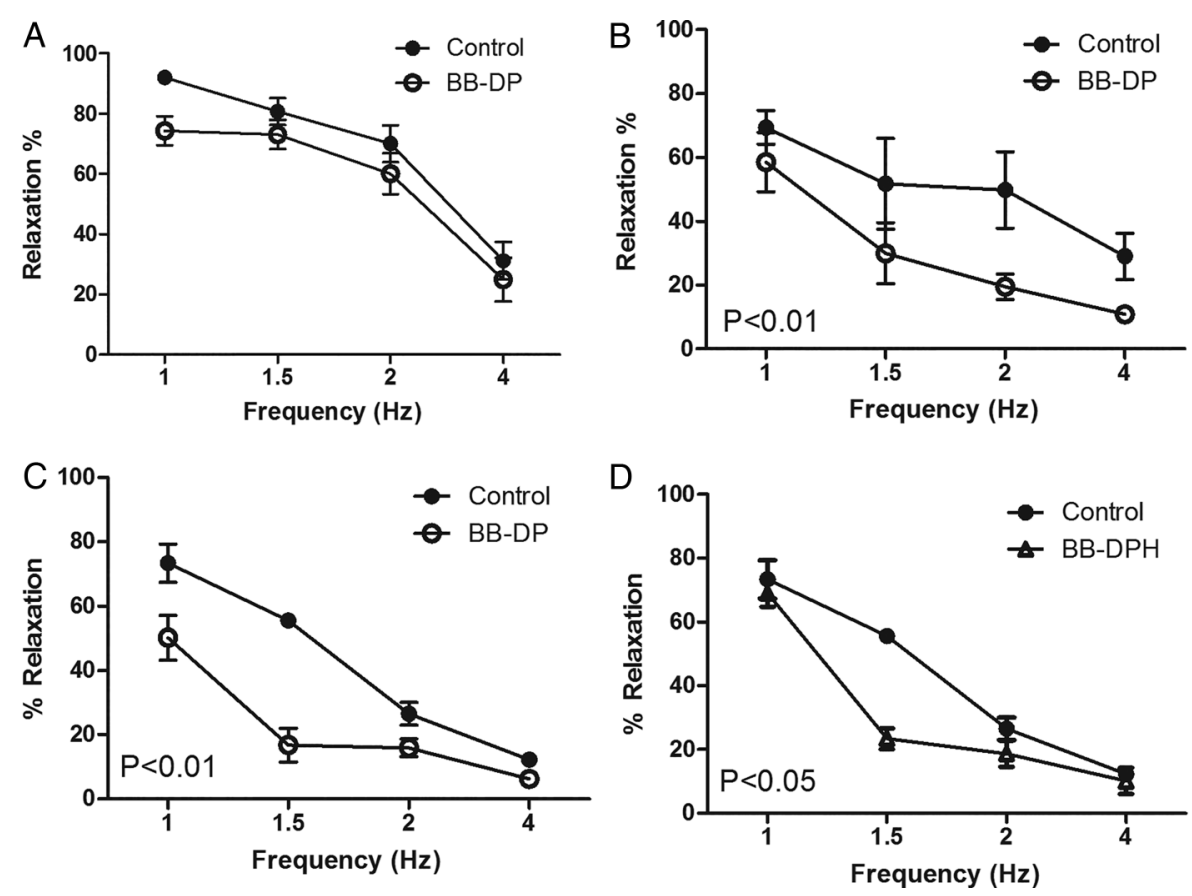

PMN cell infiltrates in the mucosa and muscle layer of this tissue. We found an increase of the total MPO activity in the mucosa and neuromuscular layer of the proximal stomach of BB-DP rats at 70 and 220 days of age in contrast to very little MPO activity at the age of 30 days. The inflammatory changes measured by MPO were confirmed by increased numbers of PMN cells in the submucosa and enteric ganglia. This was accompanied by a significantly increased amount of mast cells within the circular and longitudinal muscle layer at these time points. In addition, also hyperglycaemic animals displayed an increase of MPO activity and PMN cells, which was comparable with the values in their normoglycaemic counterparts. The absence of a significant difference between normoglycaemic and hyperglycaemic animals suggests that the inflammatory changes in the diabetic cohort are most likely not caused by the hyperglycaemic state. Nevertheless, we cannot exclude that the hyperglycaemic state also contributes to the severity of these conditions, but addressing this issue was beyond the scope of this study. The findings in BB-DP animals are reminiscent of some of the alterations found in antral biopsies of patients with paediatric FD, where an increase in mucosal mast cell counts was significantly correlated with disordered motility. ${ }^{29}$ The type of inflammation qualifies as low-grade inflammation as no scar tissue or macroscopic mucosal lesions were found in the gastric fundic biopsies of these animals.

Previous data from a trinitrobenzenesulfonic acid-induced rat model showed that inflammation can lead to impaired nitrergic function of the enteric nervous system in the small intestine. ${ }^{13}$ In this model, small intestinal motility was disrupted by the loss of nNOS protein expression as a consequence of the local inflammation. In the present study, muscle strip experiments were performed in the absence and presence of L-NAME at different time points. No differences in nitrergic function were found at 30 days, but BB rats of 70 and 220 days of age had impaired nitrergic relaxation, and this was associated with a significantly decreased total relaxation at 220 days.

Western blot analysis confirmed decreased expression of nNOS in the proximal stomach in BB-DP animals at 220 days but not at 30 and 70 days. Although nNOS expression is not altered at the age of 70 days, we did find an impaired nitrergic relaxation at this time point. This could be explained by the fact that inflammatory alterations can already impair nNOS function without a loss of the nNOS protein. ${ }^{30}$ Previous data showed that an increased expression of iNOS caused by alterations in inflammatory state correlated with the decreased expression of
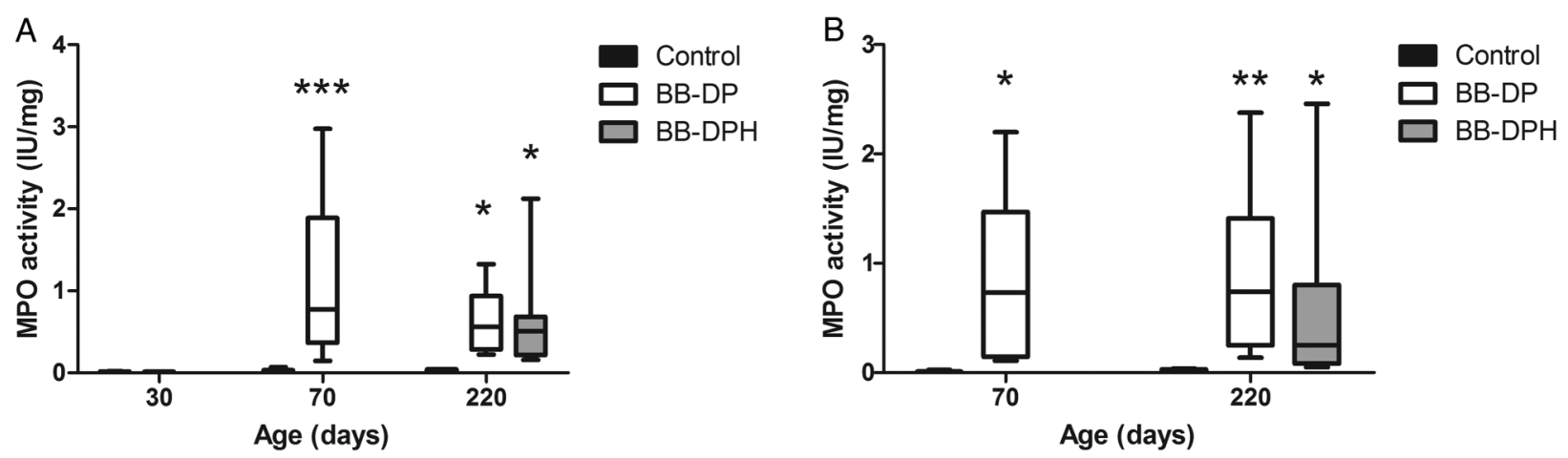

Figure 4 Myeloperoxidase (MPO) activity in mucosa and muscular layer of the gastric fundus. MPO activity is expressed in units per milligram of tissue. MPO was measured both in mucosal (A) and muscle (B) tissue and a comparison was made between control (black bars; N: 5-6), BioBreeding diabetes-prone (BB-DP) (white bars; N: 6-7) and hyperglycaemic diabetes-prone BB rats (BB-DPH) (grey bars; N: 7) done within age match groups. ${ }^{*} p<0.05,{ }^{* *} p<0.01,{ }^{* * *} p<0.001$. 
A

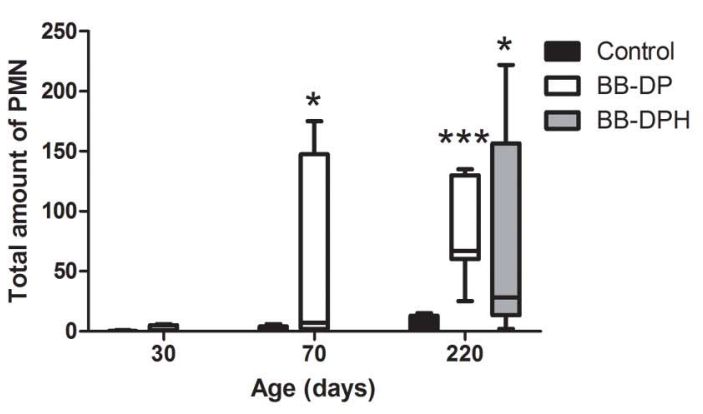

B

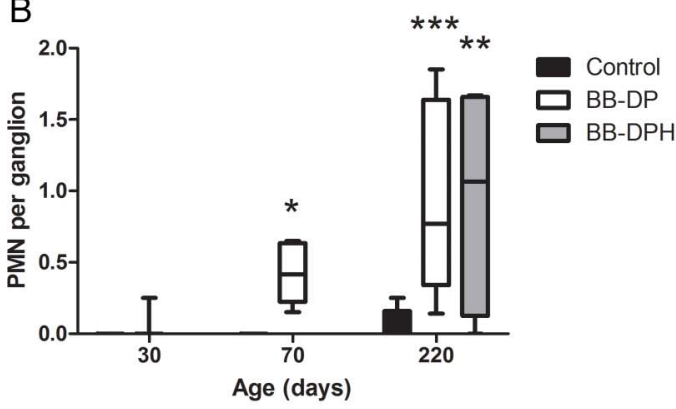

C

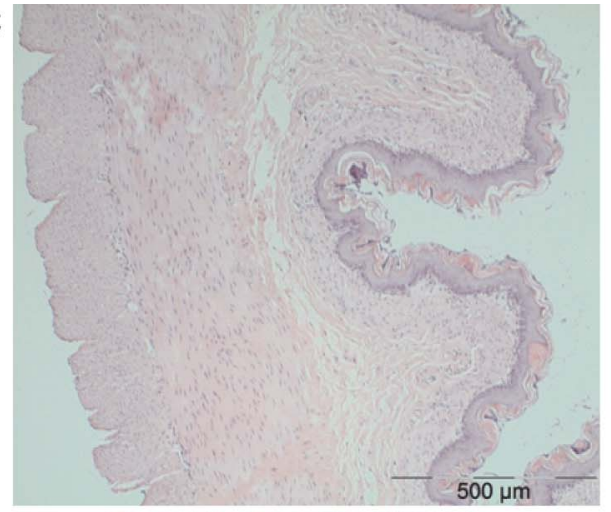

D
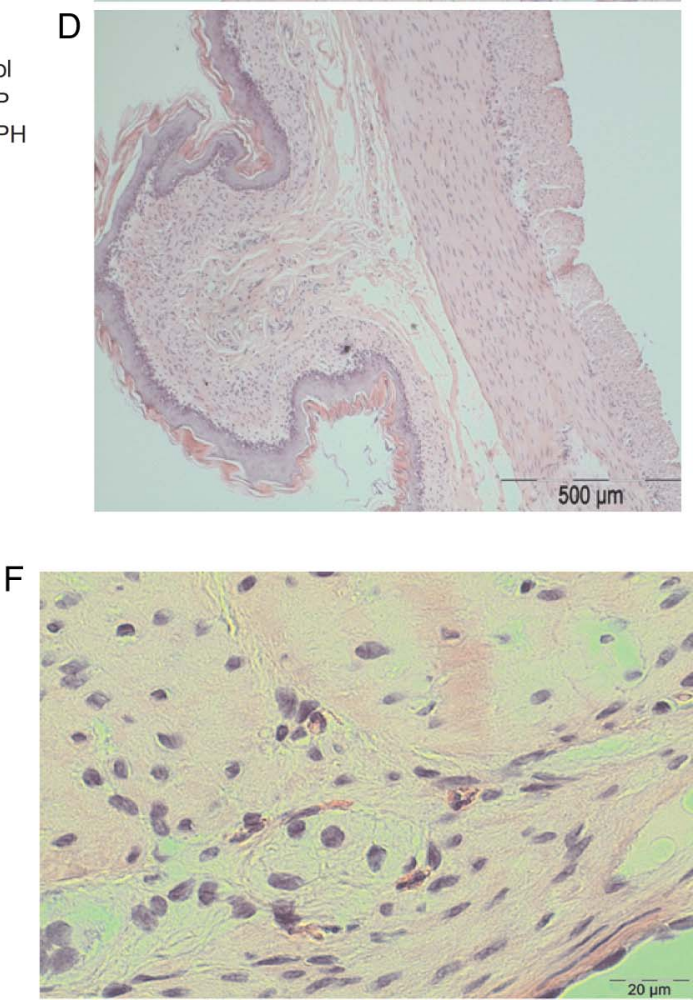

Figure 5 Histological evaluation and quantification of inflammatory changes in fundic tissue. Histological evaluation and quantification of polymorphonuclear (PMN) cells was done in H\&E-stained tissue. The total amount of PMN cells in the mucosa (A) was quantified in 10 high-power fields $(10 \times 60)$ by a single experienced pathologist. The amount of PMN in the ganglia (B) is expressed as cells per ganglion. Comparison between control (black bars; N: 5-7), BioBreeding diabetes-prone (BB-DP) (white bars; N: 5-7) and hyperglycaemic diabetes-prone BB rats (BB-DPH) (grey bars, N: 6) was done within age match groups. ${ }^{*} p<0.05$, ${ }^{* * *} p<0.001$. Representative pictures of both control (C) and BB-DP (D) rats of 220 days of age are shown after an H\&E staining. Detailed picture of ganglion from control (E) and BB-DP (F) rats, with the presence of PMN cells in the ganglion of the BB-DP rats.

Table 1 Gene expression of neuronal and inducible NOS

\begin{tabular}{|c|c|c|c|c|c|c|}
\hline Age (days) & Gene & Control & BB-DP & $p$ Value & BB-DPH & p Value \\
\hline \multirow[t]{5}{*}{70} & $n N O S-\alpha a$ & $1 \pm 0.39$ & $1.85 \pm 1.35$ & $P=0.4$ & & \\
\hline & $n N O S-\alpha b$ & $1 \pm 0.31$ & $2.10 \pm 1.11$ & $P=0.4$ & & \\
\hline & $n N O S-\alpha c$ & $1 \pm 0.12$ & $3.74 \pm 1.85$ & $P=0.3$ & & \\
\hline & $n N O S-\beta$ & $1 \pm 0.36$ & $1 \pm 0.78$ & $\mathrm{P}=0.8$ & & \\
\hline & iNOS & $1 \pm 0.12$ & $1.18 \pm 0.34$ & $P=0.9$ & & \\
\hline \multirow[t]{5}{*}{220} & $n N O S-\alpha$ a & $1 \pm 0.12$ & $0.73 \pm 0.37$ & $P=0.6$ & $0.86 \pm 0.13$ & $P=0.4$ \\
\hline & $n N O S-\alpha b$ & $1 \pm 0.16$ & $0.76 \pm 0.34$ & $\mathrm{P}=0.8$ & $0.55 \pm 0.11$ & $\mathrm{P}=0.1$ \\
\hline & $n N O S-\alpha c$ & $1 \pm 0.25$ & $0.87 \pm 0.33$ & $P=0.9$ & $1.09 \pm 0.23$ & $\mathrm{P}=0.8$ \\
\hline & $n N O S-\beta$ & $1 \pm 0.93$ & $2.40 \pm 2.48$ & $P=0.1$ & $2.17 \pm 0.49$ & $P=0.2$ \\
\hline & iNOS & $1 \pm 0.23$ & $2.5 \pm 1.4$ & $P=0.2$ & $1.39 \pm 3.5$ & $P=0.9$ \\
\hline
\end{tabular}

Gene expression of nNOS splice variants ( $n N O S \alpha$ and $n N O S \beta$ ) and iNOS was measured in control, BB-DP and BB-DPH rats of 70 and 220 days ( $n=6)$ by the use of real-time reverse transcriptase PCR. Data were normalised to the housekeeping gene, hypoxanthine phosphoribosyltransferase and expressed as fold change. Data in this table represent the fold change of BB-DP and BB-DPH rats, which is compared with a fold change of one in control rats.

BB-DP, BioBreeding diabetes prone; BB-DPH, hyperglycaemic diabetes-prone BB rats; nNOS, neuronal isoform of nitric oxide synthase; iNOS, inducible isoform of nitric oxide synthase. 

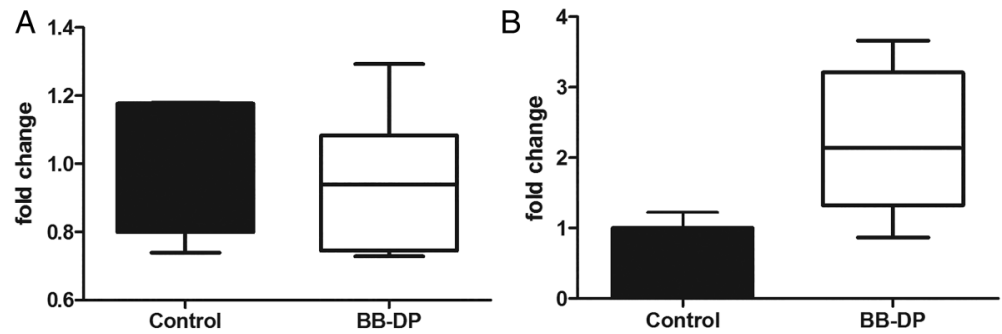

G
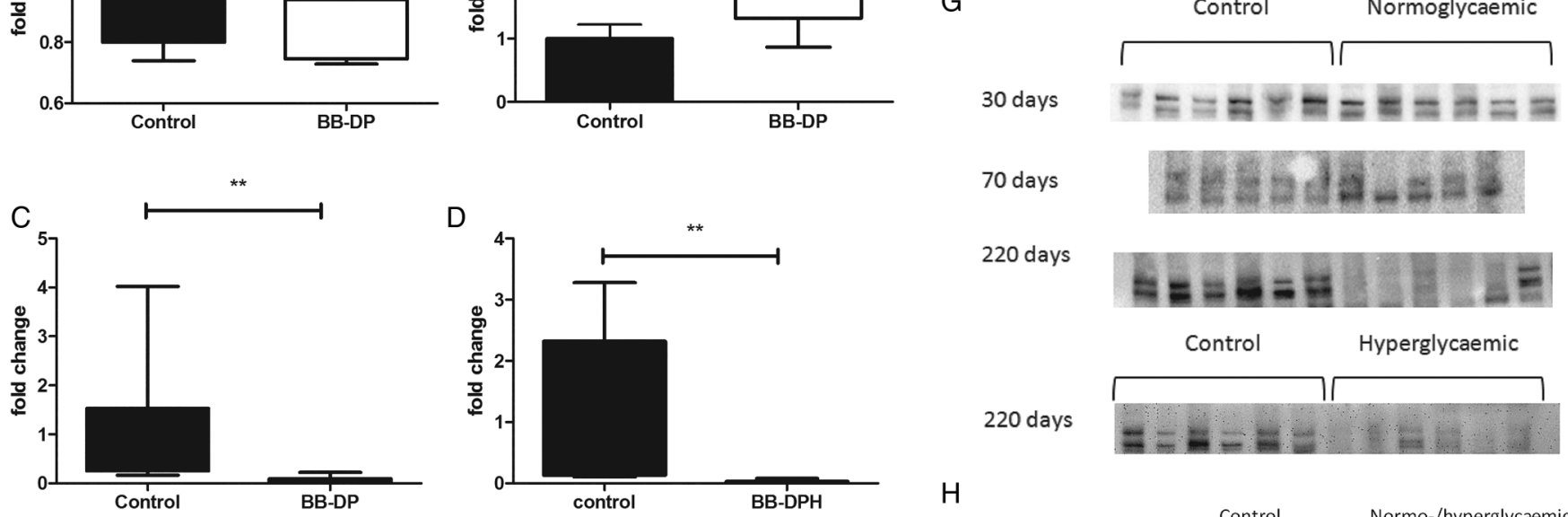

D

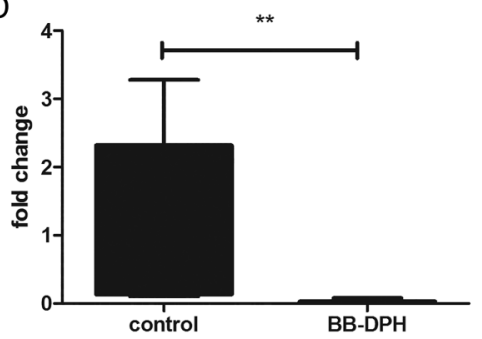

F

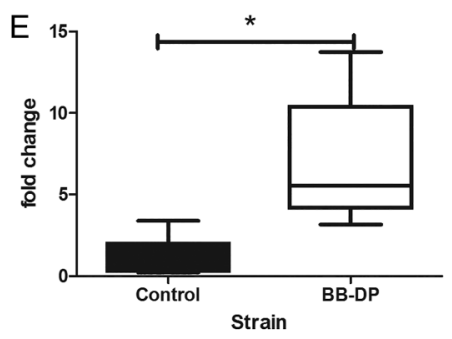

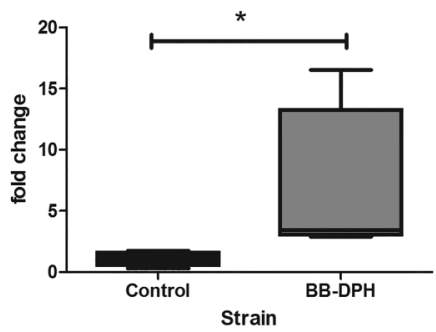

220 days

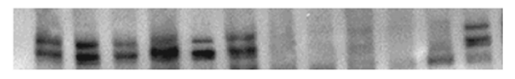

Control

Hyperglycaemic

220 days

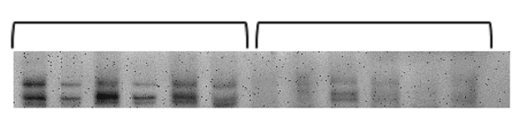

$\mathrm{H}$

Thermogycamic

iNOS Normoglycaemic

iNOS Hyperglycaemic

GAPDH

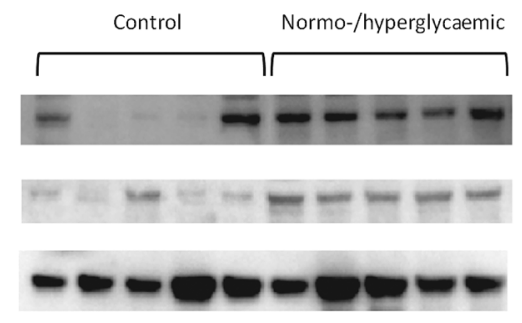

Figure 6 Western blot analysis of neuronal isoform of nitric oxide synthase (nNOS) and inducible isoform of nitric oxide synthase (iNOS) expression. Protein expression in muscular tissue of the gastric fundus was quantified by western blot analysis and compared between control (black bar) and BioBreeding diabetes-prone (BB-DP) (white bar) animals. For nNOS, this was done for all ages: (A) 30, (B) 70 and (C and D) 220 days. For iNOS, this was done for age 220 days (E and F). ( $G$ and $H)$ Representative blots. The total amount of nNOS and iNOS protein was normalised to the PGP9.5 protein and glyceraldehyde-3-phosphate dehydrogenase (GAPDH). Protein fold change was determined relative to the mean value of the control group. ${ }^{* *} \mathrm{p}<0.01$.

nNOS in animal models with colitis. ${ }^{31}$ We found a significant increase of the iNOS protein levels in both BB-DP and BB-DPH rats, which is likely to be caused by the local inflammation. Increased iNOS levels could partly explain the loss of the nNOS protein, which is underlying to the impaired gastric function.

In contrast to the expression at protein level, mRNA expression of both nNOS and iNOS was not significantly altered in our model, suggesting that changes occur mainly at the translational level. Although we cannot exclude that regulation at transcriptional and post-transcriptional level is altered, several mechanisms have been suggested by which iNOS and nNOS are increased at the protein but remain unaltered at the mRNA level. Previous data already showed that nNOS has a complex transcriptional regulation and several mRNA splice variants will translate into the same nNOS protein. ${ }^{25}$ Additionally, antisense RNA transcripts, RNA binding proteins and proteasomal activity can alter the amount of nNOS and iNOS protein expression. ${ }^{32-34}$

Both inflammatory changes and loss of nNOS are absent at the age of 30 days, which explains why nitrergic function is not yet impaired in these animals. At more advanced time points, both inflammatory changes and loss of nitrergic neurons are present and these probably represent the underlying cause for the impaired muscle function observed in the stomach.

There were no differences in PGP9.5 expression between both groups at all time points, suggesting that the total amount of neurons was preserved and argues against a non-specific neuronal damage caused by the inflammatory changes.
Kang et $a l^{35}$ described a rat model with impaired accommodation and gastric hypersensitivity after induction of gastric ulcers by injection of acetic acid in the stomach. Both mucosa and muscularis propria surrounding these ulcers were damaged, but no differences in muscle contractility were found. Although behavioural changes indicated an increased amount of discomfort and pain when accommodation was induced, changes were only described within 14 days after ulcer induction. Experiments from another research group showed that also gastric irritation with iodoacetamide in the neonatal period caused increased gastric sensitivity and impaired accommodation 8-10 weeks after induction. ${ }^{36}$ Both studies confirm that changes in gastric function can be mimicked in animal models and that inflammation can have an acute or long-term effect on gastric function. However, these models of induced damage or toxicity are less likely to reflect the clinical picture of postinfectious impaired accommodation observed in FD in humans.

In keeping with the established role of nitrergic nerves in the proximal stomach in mediating gastric accommodation, we demonstrated the presence of impaired gastric accommodation in normoglycaemic BB-DP rats. We presume that this defect was attributable to a loss of nitrergic control at the level of the gastric fundus since there was no inhibitory effect of L-NAME on accommodation. Delayed gastric emptying is unlikely to play a role in this observation since this was reported to be normal in these animals. ${ }^{14}$ As previously reported by Janssen et $a l^{23}$, changes in emptying rate (the meal we used has a half-emptying time of 80 $140 \mathrm{~min}$ ) are unlikely to influence the accommodation since this 
was measured in a short time period of only $20 \mathrm{~min}$. These observations confirm that the normoglycaemic BB-DP rat can be considered a spontaneous animal model of inflammation-related impaired gastric accommodation and provides an attractive model for further mechanistic and therapeutic studies. We were, however, not able to find a significant direct linear correlation between changes in inflammation and alterations in gastric accommodation. This can probably be explained by the fact that multiple control factors are integrated in the change in gastric accommodation as measured by the IGP technique.

Similar to previous observations, nitrergic function was also disturbed in hyperglycaemic animals compared with control rats. The underlying pathophysiology in BB-DP rats is likely to be different from those observed in the gastric fundus of hyperglycaemic BB rats, where metabolic dysfunction is more likely to be the key mechanism. ${ }^{20}$

Increased intestinal permeability and low-grade inflammation have been found in patients suffering from functional GI disorders. ${ }^{37}$ We described earlier that BB-DP rats have an increased intestinal permeability that precedes mucosal inflammation, which is followed by loss of nitrergic nerve function. ${ }^{22}$ These data suggest that increased permeability promotes local inflammation that may progress transmurally and affect nitrergic nerve function. Although these findings are in the small intestine, previous data showed that gastric permeability was also increased in the BB rat model prior to the development of diabetes. ${ }^{38}$ This suggests that an increased permeability of the stomach is a potential trigger that may lead to low-grade inflammation and loss of nitrergic neurons. However, this aspect is beyond the scope of the current study.

In summary, although an impaired nitrergic function at the level of the intestine and the antrum was previously observed in hyperglycaemic $\mathrm{BB}$ rats, we are the first to report that normoglycaemic BB-DP rats also develop impaired nitrergic motor control at the level of the myenteric plexus of the gastric fundus. This underlies the impaired gastric accommodation that was observed in the same animals. The precipitating trigger for these alterations seems to be low-grade inflammation, accompanied by an increase of mast cells and followed by decreased nNOS expression. These findings are reminiscent of previous observations in patients with postinfectious FD with impaired accommodation where an ongoing low-grade inflammation was also suggested. ${ }^{911}$ Our findings identify the BB-DP BB rat the first and promising spontaneous animal model for the study of inflammation-induced gastric motor dysfunction.

Contributors CV, TV, TM, GDH and HV contributed to the experimental work. CV and TV contributed to the statistical analysis. CV, TV, PVB, RF and JT contributed to the writing and revision of the paper.

\section{Competing interests None.}

Provenance and peer review Not commissioned; externally peer reviewed.

\section{REFERENCES}

1 Camilleri M. Integrated upper gastrointestinal response to food intake. Gastroenterology 2006;131:640-58.

2 Tack J, Demedts I, Meulemans A, et al. Role of nitric oxide in the gastric accommodation reflex and in meal induced satiety in humans. Gut 2002;51:219-24

3 Janssen $\mathrm{P}$, Vanden Berghe $\mathrm{P}$, Verschueren $\mathrm{S}$, et al. Review article: the role of gastric motility in the control of food intake. Aliment Pharmacol Ther 2011;33:880-94.

4 Tack J, Piessevaux H, Coulie B, et al. Role of impaired gastric accommodation to a meal in functional dyspepsia. Gastroenterology 1998;115:1346-52.

5 Parkman HP, Camilleri M, Farrugia G, et al. Gastroparesis and functional dyspepsia: excerpts from the AGA/ANMS meeting. Neurogastroenterol Motil 2010;22:113-33.

6 Kumar A, Attaluri A, Hashmi S, et al. Visceral hypersensitivity and impaired accommodation in refractory diabetic gastroparesis. Neurogastroenterol Motil 2008;20:635-42.
7 Tack J, Talley NJ. Functional dyspepsia-symptoms, definitions and validity of the Rome III criteria. Nat Rev Gastroenterol Hepatol 2013;10:134-41.

8 Tack J, Masaoka T, Janssen P. Functional dyspepsia. Curr Opin Gastroenterol 2011;27:549-57.

9 Tack J, Demedts I, Dehondt G, et al. Clinical and pathophysiological characteristics of acute-onset functional dyspepsia. Gastroenterology 2002;122:1738-47.

$10 \mathrm{Li} \mathrm{X}$, Chen $\mathrm{H}$, Lu H, et al. The study on the role of inflammatory cells and mediators in post-infectious functional dyspepsia. Scand J Gastroenterol 2010:45:573-81.

11 Tornblom H, Lindberg G, Nyberg B, et al. Full-thickness biopsy of the jejunum reveals inflammation and enteric neuropathy in irritable bowel syndrome. Gastroenterology 2002;123:1972-9.

12 Mizuta Y, Isomoto H, Takahashi T. Impaired nitrergic innervation in rat colitis induced by dextran sulfate sodium. Gastroenterology 2000;118:714-23.

13 Demedts I, Geboes K, Kindt S, et al. Neural mechanisms of early postinflammatory dysmotility in rat small intestine. Neurogastroenterol Motil 2006;18:1102-11.

14 Demedts I, Masaoka T, Kindt S, et al. Gastrointestinal motility changes and myenteric plexus alterations in spontaneously diabetic biobreeding rats. J Neurogastroenterol Motil 2013;19:161-70.

15 Vanormelingen C, Vanuytsel T, Masaoka T, et al. Increased Small Intestinal Permeability Precedes Mucosal Inflammation and Myenteric Ganglionitis in Non-Diabetic BB-Rats. Gastroenterology 2012;142:S810.

16 Kindt S, Boesmans W, Masaoka T, et al. Contribution of Hyperglycemia, HO- 1 Expression and Inflammation to Loss of nNOS and c-KIT Expression in the Spontaneously Diabetic Biobreeding-Rat. Gastroenterology 2009;136:A145.

17 Visser JT, Lammers K, Hoogendijk A, et al. Restoration of impaired intestinal barrier function by the hydrolysed casein diet contributes to the prevention of type 1 diabetes in the diabetes-prone BioBreeding rat. Diabetologia 2010;53:2621-8.

18 Hardin JA, Donegan L, Woodman RC, et al. Mucosal inflammation in a genetic model of spontaneous type I diabetes mellitus. Can J Physiol Pharmacol 2002;80:1064-70.

19 Takahashi T, Kojima Y, Tsunoda Y, et al. Impaired intracellular signal transduction in gastric smooth muscle of diabetic BB/W rats. Am J Physiol 1996;270:G411-17.

20 Takahashi T, Nakamura K, Itoh $\mathrm{H}$, et al. Impaired expression of nitric oxide synthase in the gastric myenteric plexus of spontaneously diabetic rats. Gastroenterology 1997; 113:1535-44.

21 Zandecki M, Vanden Berghe P, Depoortere I, et al. Characterization of myenteric neuropathy in the jejunum of spontaneously diabetic BB-rats. Neurogastroenterol Motil 2008;20:818-28.

22 Vanuytsel T, Vanormelingen $\mathrm{C}$, Van-Heel $\mathrm{H}$, et al. Impaired intestinal barrier function precedes mucosal inflammation and myenteric ganglionitis in the BB-rat. Neurogastroenterol Motil 2012;24:31.

23 Janssen $\mathrm{P}$, Nielsen MA, Hirsch I, et al. A novel method to assess gastric accommodation and peristaltic motility in conscious rats. Scand J Gastroenterol 2008;43:34-43.

24 Smits GJ, Lefebvre RA. Development of cholinergic and inhibitory non-adrenergic non-cholinergic responses in the rat gastric funds. Br J Pharmacol 1996;118:1987-94.

25 Saur D, Neuhuber WL, Gengenbach B, et al. Site-specific gene expression of nNOS variants in distinct functional regions of rat gastrointestinal tract. Am J Physiol Gastrointest Liver Physiol 2002;282:G349-58

26 Schmittgen TD, Livak KJ. Analyzing real-time PCR data by the comparative $C(T)$ method. Nat Protoc 2008;3:1101-8.

27 Meehan CJ, Fleming S, Smith W, et al. Idiopathic megacolon in the BB rat. Int J Exp Pathol 1994;75:37-42.

28 Courtois $\mathrm{P}$, Sener A, Scott FW, et al. Peroxidase activity in the intestinal tract of Wistar-Furth, BBC and BBdp rats. Diabetes Metab Res Rev 2004;20:305-14.

29 Friesen CA, Lin Z, Singh M, et al. Antral inflammatory cells, gastric emptying, and electrogastrography in pediatric functional dyspepsia. Dig Dis Sci 2008;53:2634-40.

30 Gangula PR, Maner WL, Micci MA, et al. Diabetes induces sex-dependent changes in neuronal nitric oxide synthase dimerization and function in the rat gastric antrum. Am J Physiol Gastrointest Liver Physiol 2007;292:G725-33.

31 Menchen L, Colon AL, Madrigal JL, et al. Activity of inducible and neuronal nitric oxide synthases in colonic mucosa predicts progression of ulcerative colitis. Am J Gastroenterol 2004;99:1756-64.

32 Korneev SA, Park JH, O'Shea M. Neuronal expression of neural nitric oxide synthase (nNOS) protein is suppressed by an antisense RNA transcribed from an NOS pseudogene. J Neurosci 1999;19:7711-20.

33 Aquilano K, Rotilio G, Ciriolo MR. Proteasome activation and nNOS down-regulation in neuroblastoma cells expressing a $\mathrm{Cu}, \mathrm{Zn}$ superoxide dismutase mutant involved in familial ALS. J Neurochem 2003;85:1324-35.

34 Musial A, Eissa NT. Inducible nitric-oxide synthase is regulated by the proteasome degradation pathway. J Biol Chem 2001;276:24268-73.

35 Kang YM, Lamb K, Gebhart GF, et al. Experimentally induced ulcers and gastric sensorymotor function in rats. Am J Physiol Gastrointest Liver Physiol 2005;288:G284-91.

36 Liu LS, Winston JH, Shenoy MM, et al. A rat model of chronic gastric sensorimotor dysfunction resulting from transient neonatal gastric irritation. Gastroenterology 2008;134:2070-9.

37 Vanheel H, Vicario M, Vanuytsel T, et al. Impaired duodenal mucosal integrity and low-grade inflammation in functional dyspepsia. Gut 2014;63:262-71.

38 Meddings JB, Jarand J, Urbanski SJ, et al. Increased gastrointestinal permeability is an early lesion in the spontaneously diabetic BB rat. Am J Physiol 1999;276:G951-7. 